\title{
Lithium-Ion Battery Capacity Estimation: A Method Based on Visual Cognition
}

\author{
Yujie Cheng, Laifa Tao, and Chao Yang \\ School of Aeronautic Science and Engineering, Beihang University, Beijing, China \\ Correspondence should be addressed to Laifa Tao; taolaifa@buaa.edu.cn
}

Received 18 August 2017; Accepted 16 November 2017; Published 17 December 2017

Academic Editor: Rafał Burdzik

Copyright (C) 2017 Yujie Cheng et al. This is an open access article distributed under the Creative Commons Attribution License, which permits unrestricted use, distribution, and reproduction in any medium, provided the original work is properly cited.

\begin{abstract}
This study introduces visual cognition into Lithium-ion battery capacity estimation. The proposed method consists of four steps. First, the acquired charging current or discharge voltage data in each cycle are arranged to form a two-dimensional image. Second, the generated image is decomposed into multiple spatial-frequency channels with a set of orientation subbands by using nonsubsampled contourlet transform (NSCT). NSCT imitates the multichannel characteristic of the human visual system (HVS) that provides multiresolution, localization, directionality, and shift invariance. Third, several time-domain indicators of the NSCT coefficients are extracted to form an initial high-dimensional feature vector. Similarly, inspired by the HVS manifold sensing characteristic, the Laplacian eigenmap manifold learning method, which is considered to reveal the evolutionary law of battery performance degradation within a low-dimensional intrinsic manifold, is used to further obtain a low-dimensional feature vector. Finally, battery capacity degradation is estimated using the geodesic distance on the manifold between the initial and the most recent features. Verification experiments were conducted using data obtained under different operating and aging conditions. Results suggest that the proposed visual cognition approach provides a highly accurate means of estimating battery capacity and thus offers a promising method derived from the emerging field of cognitive computing.
\end{abstract}

\section{Introduction}

Lithium-ion (Li-ion) batteries, featuring high energy density and light in weight, are becoming more and more popular for various applications, especially in the field of aerospace and electric vehicles [1-3]. Thus, the majority of existing studies focus on ways to improve the performance of Li-ion batteries. Battery capacity, which is regarded as an important indicator of the battery performance, is highly affected by various internal and external mechanisms such as ambient temperature, aging, and usage patterns; these factors cause battery performance to gradually fade over time. Therefore, available battery capacity needs to be accurately estimated for reliability purposes and for the proper management of battery use [4].

Recent studies have reported a variety of approaches to estimating the capacity of Li-ion batteries. Most of the existing approaches are model-based methods, including electrochemical [5], equivalent circuit-based [6], and analytical $[7,8]$ models. These models are mostly based on complex physical and chemical processes that take into account the dynamic behavior of batteries [9-11], and the estimation performance is highly dependent on the accuracy of the models. In particular, these types of models are usually difficult to establish given the restrictions on acquisition of knowledge of the electrochemical parameters, aging mechanisms, and properties of batteries [12]. Moreover, these models are individually dependent on the specific type of battery in terms of production processes, electrolytes, and anode and cathode materials. State-of-charge- (SOC-) opencircuit-voltage- $(\mathrm{OCV}-)$ based methods for in-cycle capacity estimation are widely applied in many real-world applications $[13,14]$. However, the SOC-OCV-based methods rely on accurate SOC and OCV values, which are usually highly time consuming to obtain $[10,15]$. Regardless of which modelling methods are used to model the battery state, the laboratory determined battery charging and discharging characteristics under different operating conditions are a source of knowledge about battery behavior. In some applications, these original data stored as discrete values are employed to create a lookup table database on the charge status of the master 
battery. However, using this kind of method for Li-ion battery capacity estimation, amounts of experiments need to be performed under various operating conditions for a whole lifetime to obtain battery capacities in different life states under different operating conditions. Otherwise, database-based method will have a low accuracy with a rough database. Tao et al. [16] proposed a capacity estimation method for Li-ion battery based on similarity recognition of online data curves, which can be considered as an intelligent method based on database. Even though this method achieves a high accuracy, it takes a lot of time looking for the most similar data curve contained in the database, which restricts its real application.

A new geometric-based method was proposed in [17]; this method differs from those aforementioned and appears to be an extension of the traditional constant currentconstant voltage method [18]. It estimates battery capacity by combining differential geometry and four geometric features that are sensitive to capacity fade. The four geometric features are extracted from the charging current (CC) and discharging voltage (DV) curves, including the time duration of the constant voltage $(\mathrm{CV})$ curve, maximum radius of curvature of the CV stage, area under the CV curve, and slope of the voltage curve in the early stage of the discharge process. Experimental results provided in their paper demonstrate the effectiveness of the geometric-based method.

The nature of the geometric-based method aims to introduce differential geometry theory and traditional geometric features into battery capacity estimation. Inspired by the work in [17], we attempt to introduce other leading-edge interdisciplinary methods to battery capacity estimation, avoiding complicated analyses of physical-chemical processes and achieving an accurate cognition of degradation processes, thereby further enhancing the effectiveness and accuracy of battery capacity estimation.

Cognitive science is an interdisciplinary study that consists of multiple research disciplines, including psychology, artificial intelligence, philosophy, neuroscience, linguistics, and anthropology. It includes research on intelligence and behavior, especially focusing on how information is represented, processed, and transformed within nervous systems and machines [19]. Cognitive science is a large field and covers a wide array of topics relating to cognition such as language processing, artificial intelligence, and visual and auditory cognition. Among these topics, visual cognition has become the focus of many studies in cognitive science and is becoming a significant topic of interest in the twenty-first century [20]. In recent years, countries around the world have invested heavily to support research in visual cognition. In the US, the Defense Advanced Research Projects Agency launched a special research program in 2007 named "Cognitive Computing," under which visual cognition is a key research target. In Japan, experts in the field of computer vision have been brought into the "Brain Plan" over the past decade to promote the interdisciplinary studies of brain cognitive science and visual cognition. The National Natural Science Foundation Committee of China initiated a major research project in 2008 called "Cognitive Computing Based on Visual and Auditory Information"; its purpose is to establish a new computational method based on human visual and auditory cognitive mechanisms, thereby providing new ideas for image understanding and voice processing. Today, computing methods based on visual cognition have received extensive attention and are widely used in face recognition [21], image fusion [22], texture classification [23], and so forth. However, in the field of Li-ion battery capacity estimation, methods based on visual cognition have rarely been reported. Motivated by this, we attempt to transform CC values and DV values into a two-dimensional image and thus to further advance battery capacity estimation using a visual cognition method.

Essentially, visual cognition is a kind of bionic science; that is, it deals with the recognition of objects based on the characteristics of the human visual system (HVS). One of the well-known characteristics of the HVS is the multichannel characteristic (MCC), meaning that there are multiple spatial-frequency channels in the processing of pictorial information in the HVS, each of which further involves different number of orientation components depending upon a predetermined setting of series [24]. In this study, the authors employ MCC to extract degradation feature information from CC and DV data, which is the core of this study and also the difference distinguishing our method from other existing methods, including that in [17]. Another noted HVS characteristic is the manifold sensing characteristic (MSC). In 2000, articles published in Science pointed out that (1) visual information is stored as a manifold of stable neural-activity patterns in the brain, and (2) manifold learning methods can identify meaningful low-dimensional structures in highdimensional data [25-27]. Therefore, this study utilizes the manifold learning to construct a low-dimensional intrinsic manifold, which can not only reveal the capacity degradation law that is contained in the extracted features but also reduce the computation required. Therefore, this study attempts to introduce visual cognition into Li-ion battery capacity estimation in order to establish a systematic method for capacity estimation based on MCC and MSC.

This paper is organized as follows: Section 2 describes the two HVS properties of interest, namely, MCC and MSC, as well as the corresponding computing methods derived from them, primarily NSCT and the Laplacian eigenmap (LE). Geodesic distance is also introduced, which is used in the estimation of battery capacity. Section 3 presents the entire method for battery capacity estimation based on visual cognition, including descriptions of the experimental data, image transformation, feature extraction, and capacity calculation. Typical data from NASA battery data sets are utilized to verify the proposed method; the results are reported in Section 4. Finally, Section 5 concludes the paper.

\section{Related Theories}

\subsection{MCC of the HVS and NSCT}

2.1.1. MCC and Contourlet Transform. The HVS is a crucial tool by which human beings understand and comprehend the natural world. It has been verified that the HVS possesses the ability to capture the essential information of a natural scene using a minimal number of active visual cells [28]. 
The receptive fields in the visual cortex are accordingly characterized as being localized, oriented, and bandpass [29]. Therefore, it is suggested that, for an image representation to be efficient, it should have the properties of being local, directional, and of multiresolution.

The contourlet transform (CT) proposed by Do and Vetterli [28] matches the MCC of the HVS well. It is composed of a Laplacian pyramid (LP) and a directional filter bank (DFB), where the LP is employed to capture the point discontinuities and the DFB is utilized to link point discontinuities to linear structures. The CT gives a flexible multiresolution, local, and directional image expansion using contour segments; thus, it can represent edges and other singularities along curves very efficiently. Unfortunately, however, the CT lacks shift invariance because of the downsampling and upsampling in both the LP and the DFB. In particular, downsampling of a filtered image may result in low-pass and high-pass frequency aliasing. These shortcomings limit the use of CT in many applications $[22,30]$.

2.1.2. NSCT Theory. To eliminate the frequency aliasing of $\mathrm{CT}$ and enhance its directional selectivity and shift invariance, da Cunha et al. [31] proposed a shift-invariant version based on non-subsampled pyramid filter banks (NSPFBs) and non-subsampled directional filter banks (NSDFBs), as shown in Figure 1(a) [31, 32].

The non-subsampled contourlet transform (NSCT), as a representative method related to the MCC, can be used to decompose an image (e.g., as transformed from a charge current or discharge voltage curve) into multiple spatialfrequency channels (a set of narrow-band frequencies), each of which further involves different number of orientation components depending upon a predetermined setting for each channel.

In NSCT, the multiscale property is obtained from a shift-invariant filtering structure that achieves a subband decomposition similar to that of the LP. The process can be implemented using two-channel non-subsampled twodimensional (2D) filter banks. Figure 1(b) illustrates the nonsubsampled pyramid decomposition with $J=3$ stages. Such an expansion is conceptually similar to the one-dimensional (1D) non-subsampled wavelet transform computed with the à trous algorithm. The filters for the next stage are obtained by upsampling the filters of the previous stage with the sampling matrix:

$$
D=2 I=\left[\begin{array}{ll}
2 & 0 \\
0 & 2
\end{array}\right]
$$

which gives the multiscale property without the need for additional filter design. On the $j$ th decomposition, the ideal frequency support of the low-pass filter is $\left[-\left(\pi / 2^{j}\right),\left(\pi / 2^{j}\right)\right]^{2}$. Correspondingly, the ideal support of the high-pass filter is the complement of the low-pass filter, namely, the region $\left[-\left(\pi / 2^{j-1}\right),\left(\pi / 2^{j-1}\right)\right]^{2} \backslash\left[-\left(\pi / 2^{j}\right),\left(\pi / 2^{j}\right)\right]^{2}$. The equivalent filters of a $J$-level cascading NSPFB are given by

$$
H_{n}^{\mathrm{eq}}(z)= \begin{cases}H_{1}\left(z^{2^{n-1} I}\right) \Pi_{j=0}^{n-2} H_{0}\left(z^{2^{j} I}\right), & 1 \leq n \leq J \\ \prod_{j=0}^{n-2} H_{0}\left(z^{2^{j} I}\right), & n=J+1,\end{cases}
$$

where $H_{0}(z)$ and $H_{1}(z)$ represent the low-pass filter and the corresponding high-pass filter, respectively, at the first stage [32].

The DFB is constructed by combining critically sampled two-channel fan filter banks and resampling operations. This results in a tree-structured filter bank that splits the $2 \mathrm{D}$ frequency plane into directional wedges. By switching off the downsamplers/upsamplers in each two-channel filter bank in the DFB tree structure and upsampling the filters accordingly, the NSDFB is obtained. In this manner, a tree composed of two-channel NSDFBs can be obtained. Figure 1(c) illustrates a four-channel decomposition [32]. The upsampled fan filters $U_{j}\left(z^{\mathrm{Q}}\right)(j=0,1)$ have checkerboard frequency support, where $Q$ is the quincunx matrix:

$$
Q=\left[\begin{array}{cc}
1 & -1 \\
1 & 1
\end{array}\right] .
$$

The four-channel directional decomposition can be obtained when filters $U_{j}\left(z^{\mathrm{Q}}\right)$ are combined with the fan filters $U_{i}(z)(i=0,1)$. The equivalent filter in each channel $U_{k}(z)(k=0,1,2,3)$ can be given as follows:

$$
U_{k}(z)=U_{i}(z) U_{j}\left(z^{\mathrm{Q}}\right) .
$$

After $J$-level NSCT decomposition, one low-pass subband image and $\sum_{j=1}^{J} 2^{l_{j}}$ bandpass directional subband images can be obtained, all of which have the same size as the input image. Here, $l_{j}$ is the directional decomposition level at the $j$ th scale.

As described above, the core of NSCT is the filter design in the two-channel NSPFB and NSDFB. NSCT not only retains the characteristics of CT but also has the important property of shift invariance. Thus, this study employs NSCT to extract features from the CC and DV values of a Li-ion battery.

\subsection{MSC of the HVS and LE}

2.2.1. MSC and Manifold Learning. As we look at an object with conditions such as scale and illumination changing, the signals carried from the eyes to the brain by the millions of axons in the optic nerve are constantly in flux. Nevertheless, we are able to recognize that these changing signals are produced by the same object. This phenomenon was studied by Seung and Lee, who proposed a hypothesis that a visual memory is stored as a manifold of stable states or a continuous attractor [25]. Images of the same object with changes in scale, illumination, and other variable factors lie on a lowdimensional manifold, whereas images of different objects form different manifolds. From the perspective of cognitive psychology, the cognitive process of object identification 

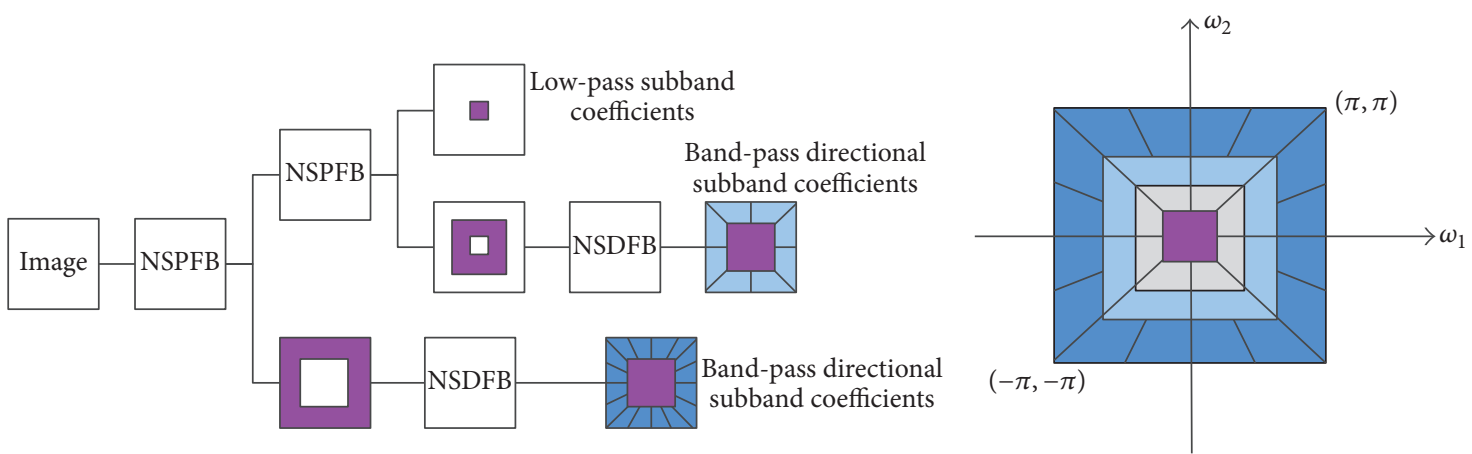

(a)
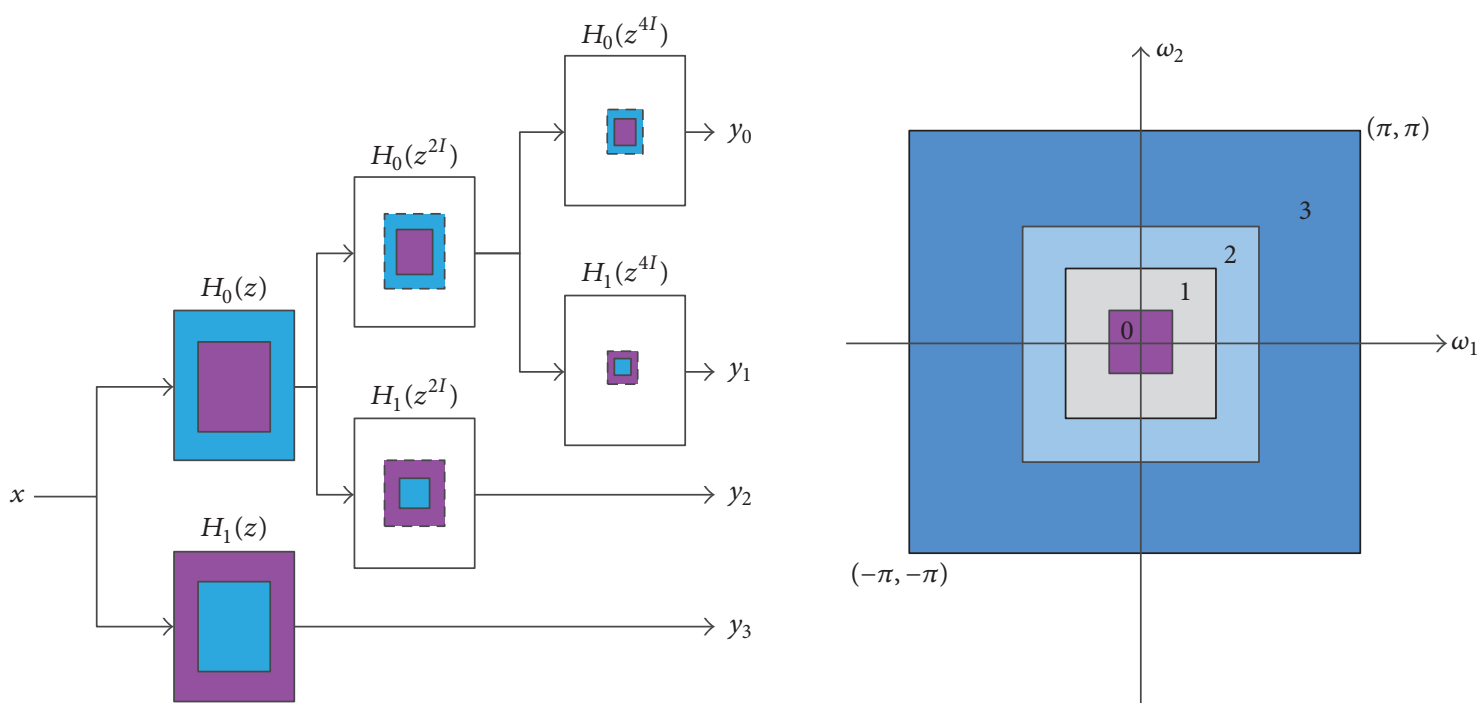

(b)
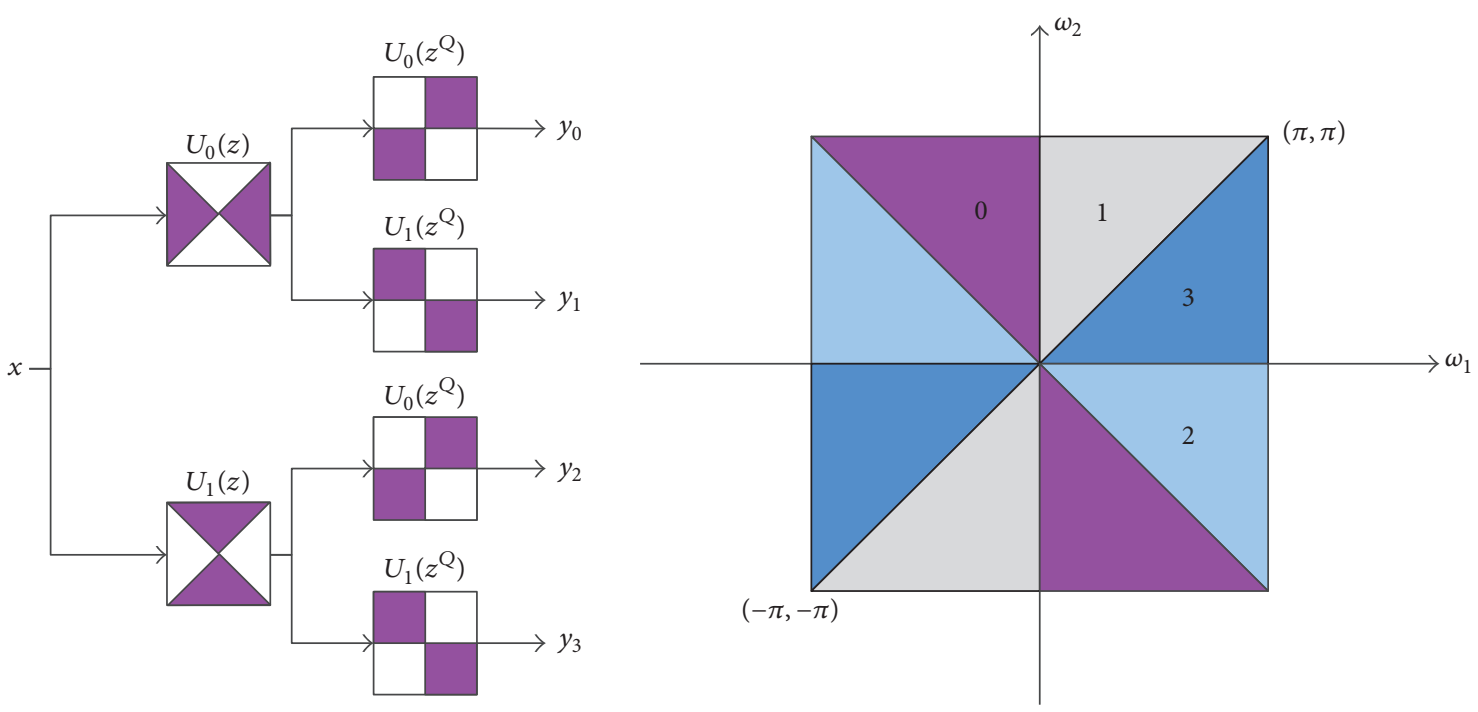

(c)

FIGURE 1: Schematic diagram of non-subsampled contourlet transform (a), three-stage non-subsampled pyramid decomposition (b), and four-channel NSDFB constructed with two-channel fan filter bank (c). 
is the recognition of different low-dimensional manifolds embedded in the high-dimensional visual information. That is, the HVS has the ability to sense the manifold hidden in the brain. This characteristic of the HVS is called MSC. Similar to the MSC of the HVS, manifold learning can find meaningful low-dimensional structures hidden within high-dimensional observations; this is attracting increasing attention from scholars.

Manifold learning, also known as nonlinear dimensionality reduction, is a widely accepted method that embeds highdimensional samples into low-dimensional feature space by preserving some local or global geometric structures [33]. Many manifold learning approaches have been proposed, such as isometric mapping [26], locally linear embedding [27], Laplacian eigenmaps [34], and Hessian eigenmaps [35]. Among these approaches, the Laplacian eigenmap (LE) is a kind of spectral graph method; this has received considerable attention from the machine learning community. In this study, the LE is employed to establish a low-dimensional intrinsic manifold and carry out the dimensionality reduction.

2.2.2. Theory of $L E$. LE is a typical graph-based dimensionality reduction technique. The basic mathematical notion of LE can be summarized as follows.

Assume that a $d$-dimensional manifold $M^{d}$ (nominated as output space) embedded in an $m$-dimensional space $\alpha_{N} \epsilon$ $R^{m}$ (nominated as input space, $d<m$ ) can be described by a function:

$$
f: C \subset M^{d} \longrightarrow R^{m},
$$

where $C$ is a compact subset of $M^{d}$ with open interior. A set of data points $\alpha_{1}, \ldots, \alpha_{N}$, where $\alpha_{i} \in R^{m}$, are sampled with noise from the intrinsic manifold $M^{d}$; the relationship can be represented as follows:

$$
\alpha_{i}=f\left(\beta_{i}\right)+\xi_{i}, \quad i=1, \ldots, N
$$

where $\xi_{i}$ denotes noise. LE can be recognized as follows: the original data set $\alpha_{i}$ 's in the higher dimensional manifold $R^{m}$ are mapped (nonlinearly) to the data point $\beta_{i}$ 's in the estimation of the unknown lower dimensional manifold $M^{d}$, with $d<m$ [36].

Given a set of $N$ multivariate observations, for arbitrary point $A \in M^{d}$ with $k$ nearest neighborhoods, a weighted adjacency graph $G=(V, E)$ can be constructed consisting of $N$ nodes and a set of edges connecting neighboring points. We consider the problem of mapping the weighted graph $G$ to a line such that the connected points stay as close together as possible. Let $\mathbf{y}=\left(y_{1}, y_{2}, \ldots, y_{N}\right)^{T} \mathbf{x}=\left(x_{1}, x_{2}, \ldots, x_{N}\right)^{T}$, where $x_{i}, y_{i} \in R$ is a coordinate value of the $i$ th point in $R^{m}$ and $M^{d}$. A reasonable map is to choose $y_{i}$ 's $\in R$ to minimize $\sum\left(y_{i}-y_{j}\right)^{2} W_{i j}$ under the appropriate constraints. To avoid the heavy penalties that can occur if the neighboring points $x_{i}$ and $x_{j}$ are mapped far apart, the minimization is an attempt to ensure that if points $x_{i}$ and $x_{j}$ are close, then $y_{i}$ and $y_{j}$ will be close as well. As a result, for any $\mathbf{y}$, we have

$$
\frac{1}{2} \sum_{i, j}\left(y_{i}-y_{j}\right)^{2} W_{i j}=\mathbf{y}^{T} L \mathbf{y},
$$

where $L=D-W$ is the Laplacian matrix, which is positive semidefinite. Notably, $W_{i j}$ is symmetric, and $D_{i i}=\sum_{j} W_{i j}$. Thus, $\sum_{i, j}\left(y_{i}-y_{j}\right)^{2} W_{i j}$ can be written as

$$
\begin{aligned}
& \sum_{i, j}\left(y_{i}^{2}+y_{j}^{2}-2 y_{i} y_{j}\right) W_{i j} \\
& \quad=\sum y_{i}^{2} D_{i i}+\sum y_{j}^{2} D_{j j}-2 \sum y_{i} y_{j} W_{i j}=2 \mathbf{y}^{T} L \mathbf{y} .
\end{aligned}
$$

Therefore, the minimization problem reduces to finding $\arg \min _{\mathbf{y}^{T} D \mathbf{y}=1} \mathbf{y}^{T} L \mathbf{y}$.

The constraint $\mathbf{y}^{T} D \mathbf{y}=1$ removes an arbitrary scaling factor in the embedding. Matrix $D$ provides a natural measure on the graph vertex. The larger $D_{i i}$ is, the more important the vertex will be. In (7), $L$ is shown as a positive semidefinite matrix, and the vector $\mathbf{y}$ that minimizes the objective function is given by the minimum eigenvalue solution to the generalized eigenvalue problem $L \mathbf{y}=\lambda D \mathbf{y}$ with an additional

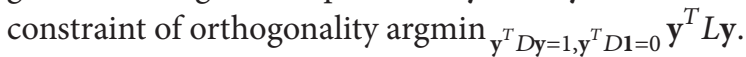

More generally, the embedding is given by the $N \times d$ matrix $Y=\left[\mathbf{y}_{1}, \mathbf{y}_{2}, \ldots, \mathbf{y}_{d}\right]$, where the $i$ th row, denoted by $Y_{i}^{T}$, provides the embedding coordinates of the $i$ th vertex. Similarly, we need to minimize

$$
\sum_{i, j}\left\|Y_{i}-Y_{j}\right\|^{2} W_{i j}=\operatorname{tr}\left(Y^{T} L Y\right) .
$$

This condition reduces to finding [37]

$$
Y_{\text {opt }}=\underset{Y^{T} D Y=1}{\operatorname{argmin}} \operatorname{tr}\left(Y^{T} L Y\right) .
$$

2.2.3. Time Window for Mapping Updating. The fixed set of data from high-dimensional space is mapped to lowdimensional space by LE through the mapping $g=f^{-1}$. Therefore, one can receive a corresponding low-dimensional point through the mapping when given an arbitrary point in the high-dimensional space. Considering in practice, new data are often collected and new features can be obtained in the $R^{m}$ space, we need to update the mapping provided by LE to adjust to the new incoming data. Thus, a general method, the so-called "time window," is proposed, which can be set as one incoming point or any other number of incoming points with regard to a real-world application. When the number of new incoming points reaches the fixed "time window," a new updated mapping is derived.

2.3. Geodesic Distance. In mathematics, particularly differential geometry, a geodesic is a generalization of the notion of a "straight line" to curved spaces [38]. If this connection is the Levi-Civita connection induced by a Riemannian metric, then the geodesics are (locally) the shortest path between 
TABLE 1: Typical data under different operating conditions.

\begin{tabular}{lcccccc}
\hline Label number & $\mathrm{AT}\left({ }^{\circ} \mathrm{C}\right)$ & $\mathrm{CC}(\mathrm{A})$ & DC $(\mathrm{A})$ & EOD $(\mathrm{V})$ & IC $(\mathrm{Ah})$ & EOLC $(\%)$ \\
\hline$\# 5$ & 24 & 1.5 & 2 & 2.7 & 3.8565 & 30 \\
$\# 7$ & 24 & 1.5 & 2 & 2.2 & 1.8911 & 1.8447 \\
$\# 29$ & 43 & 1.5 & 4 & 2.0 & 12.61 \\
$\# 54$ & 4 & 1.5 & 2 & 2.2 & 1.1665 & 30 \\
\hline
\end{tabular}

Note. AT, CC, DC, EOD, IC, and EOLC denote ambient temperature, charge current, discharge current, end-of-discharge, initial capacity, and end-of-life criteria (ratio of faded capacity to initial capacity), respectively.

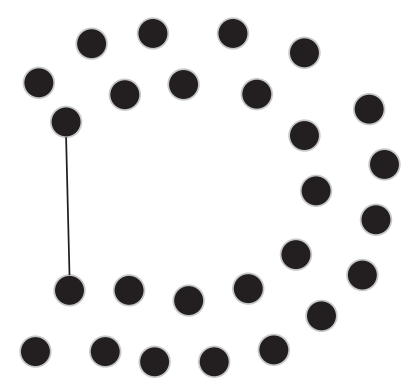

Euclidean distance

(a)

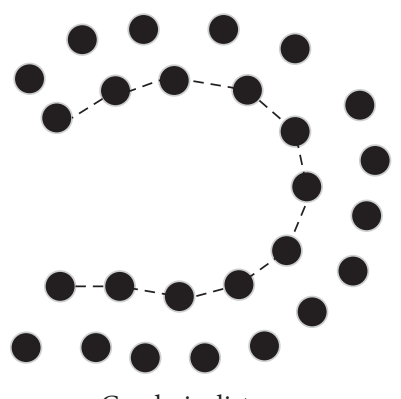

Geodesic distance

(b)
FIgure 2: Comparison between Euclidean distance and geodesic distance.

points in the space. Thus, the geodesic distance is expected to unfold highly folded, twisted, or curved nonlinear manifolds [39].

Figure 2(a) shows the shortest path measured by Euclidean distance. According to this metric, two points on opposite sides of the horseshoe appear to be deceptively close. Figure 2(b) shows the shortest path measured by geodesic distance. In this case, the two points on opposite sides of the horseshoe are not neighbors according to the geodesic distance [39].

In this study, the geodesic distance is adopted as the geometrical metric of battery capacity on the manifold constructed by LE.

\section{Method for Estimation of Li-Ion Battery Capacity Based on Visual Cognition}

3.1. Description of the NASA Li-Ion Battery Experimental Data. The data used in this study were obtained from a custom-built battery setup at the NASA Ames Prognostics Center of Excellence. The experiments were conducted through three different operational profiles (charge, discharge, and impedance) at ambient temperature (AT) conditions. Charging is performed in a constant charge current mode at $1.5 \mathrm{~A}$ until the battery voltage reaches $4.2 \mathrm{~V}$ and continues in a constant voltage mode until the charge current drops to $20 \mathrm{~mA}$. The discharge runs are stopped at different end-of-discharges (EODs). The experiments are conducted until the capacity decreases to specified end-of-life criteria (EOLC).

To validate the efficiency of the proposed approach, the typical data were selected (\#5, \#7, \#29, and \#54, which were also used in $[16,17])$ and described in Table 1. From Table 1, it can be seen that these data have the same charge current of $1.5 \mathrm{~A}$ but generally exhibit different ATs $\left(24^{\circ} \mathrm{C}, 43^{\circ} \mathrm{C}\right.$, or $4^{\circ} \mathrm{C}$ ), discharge currents (DCs; 2 A or $4 \mathrm{~A}$ ), EODs (from $2.0 \mathrm{~V}$ to $2.7 \mathrm{~V}$ ), initial capacities (ICs; from $1.1665 \mathrm{Ah}$ to $1.8911 \mathrm{Ah}$ ), and EOLCs (30\% or $12.61 \%)$.

\subsection{Image Transformation of CC or DV Values for Visual Cognition}

3.2.1. Image Transformation Method. The real state of an arbitrary battery can be identified by charging or discharging it. Consequently, the CC and DV curves obtained from the charging and discharging processes can directly reflect the real state of the battery. To discover the performance degradation law contained in these curves, the CC and DV curves for each cycle are transformed into an image for the following visual cognition. First, the CC and DV values over a lifetime of full cycles are uniformly normalized according to the linear normalization equation: $y=(x-$ MinValue)/(MaxValue - MinValue), where $x$ is the original $\mathrm{CC}$ or DV value, $y$ is the normalized value, and MinValue and MaxValue are the minimum and maximum $\mathrm{CC} / \mathrm{DV}$ values over the lifetime of full cycles, respectively. The normalized data points are then arranged into an $M \times N$ matrix, as shown in Figure 3. If we consider the normalized amplitude of each sample as a pixel value of an image, then the $M \times N$ matrix becomes an $M \times N$ image. The following principles are used to ensure the quality of the transformed images: (1) the transformed images should retain the most useful information of each charge/discharge cycle; (2) the CC and DV data that differ significantly from those of other cycles should be eliminated; (3) the images built based on the CC and DV data of each cycle should have the same size. To adhere to these principles, the CC and DV data of each charge/discharge cycle need to be selected and processed.

3.2.2. Data Selection and Processing. To some extent, the quality of the image transformation directly affects the visual cognition results. Therefore, appropriate selection and processing of the CC and DV data are essential to ensure high-quality transformed images.

Our experiments collect two kinds of CC/DV data containing the most useful information: (1) CC data under the constant voltage charging stage; (2) DV data during the discharging process. The following data are discarded: (A) abnormal data, (B) CC data under the constant current charging stage, $(C)$ sensitive voltage data from the early stages of discharge, and (D) voltage recovery data; these are shown in Figure 4. 


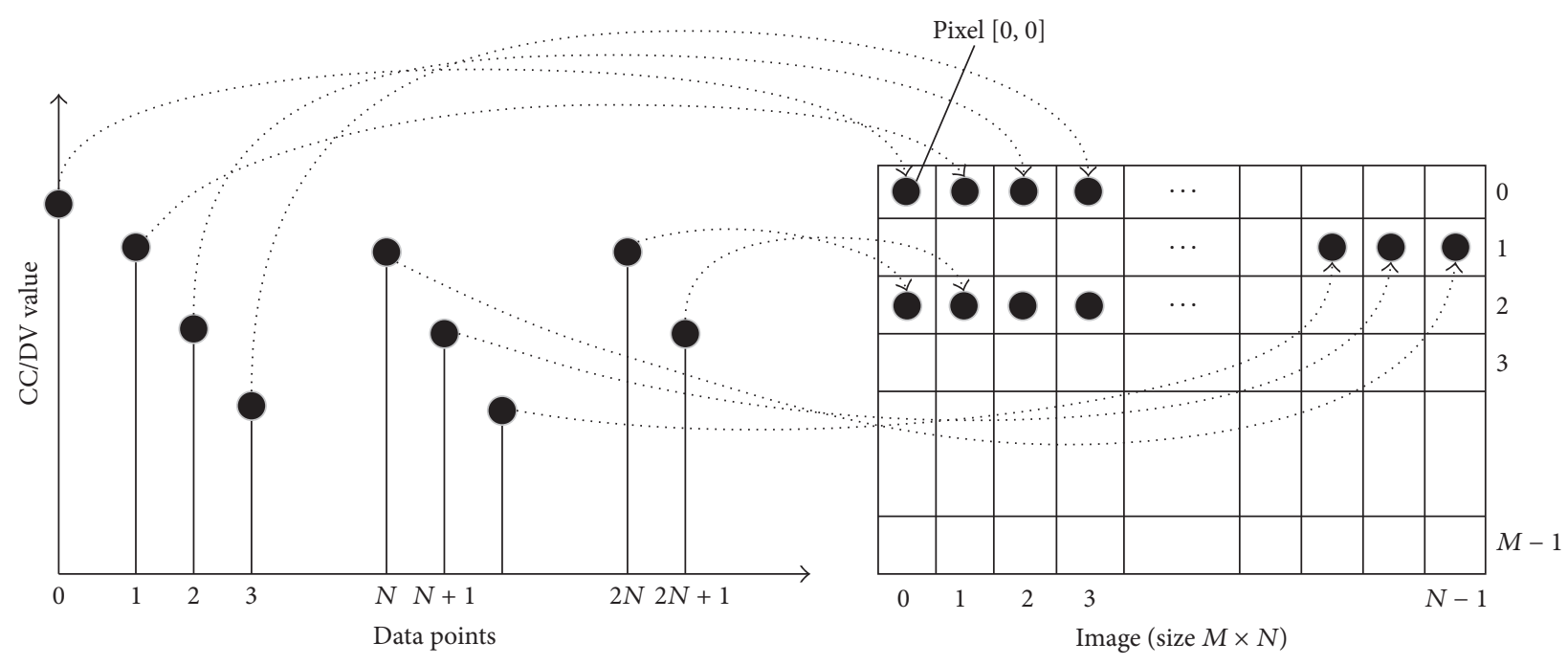

FIGURE 3: Image transformation scheme based on CC/DV values.
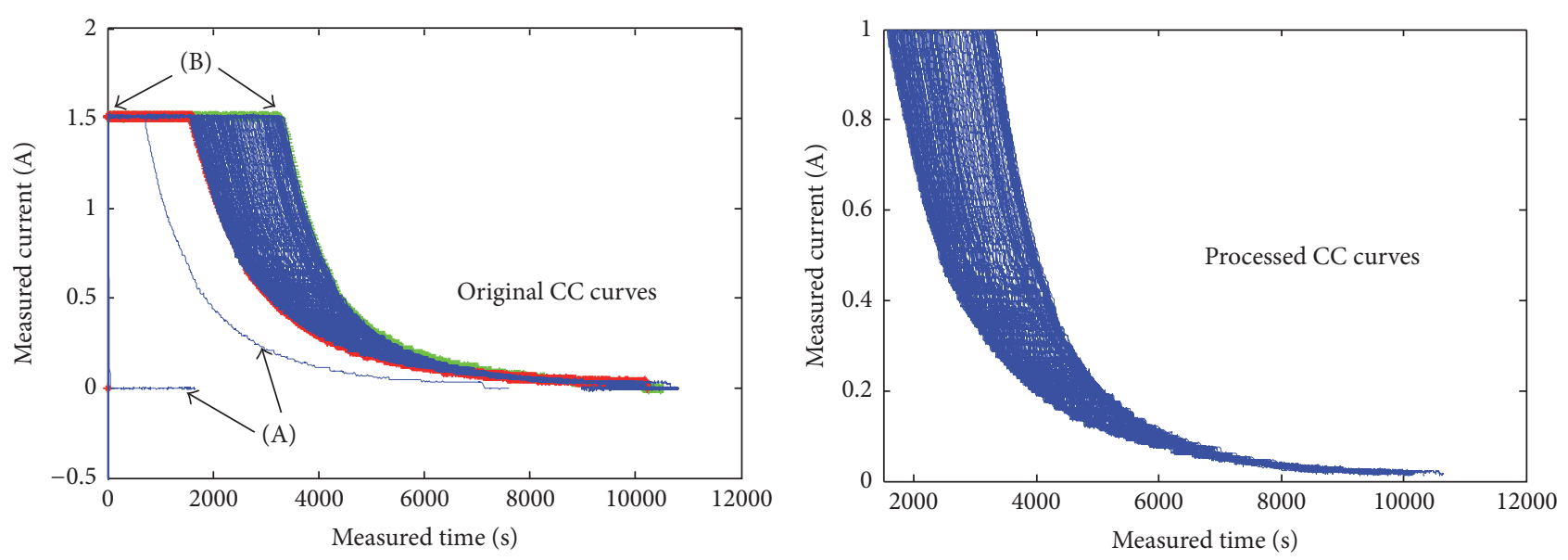

+ The first curve

* The last curve

(a)

(b)
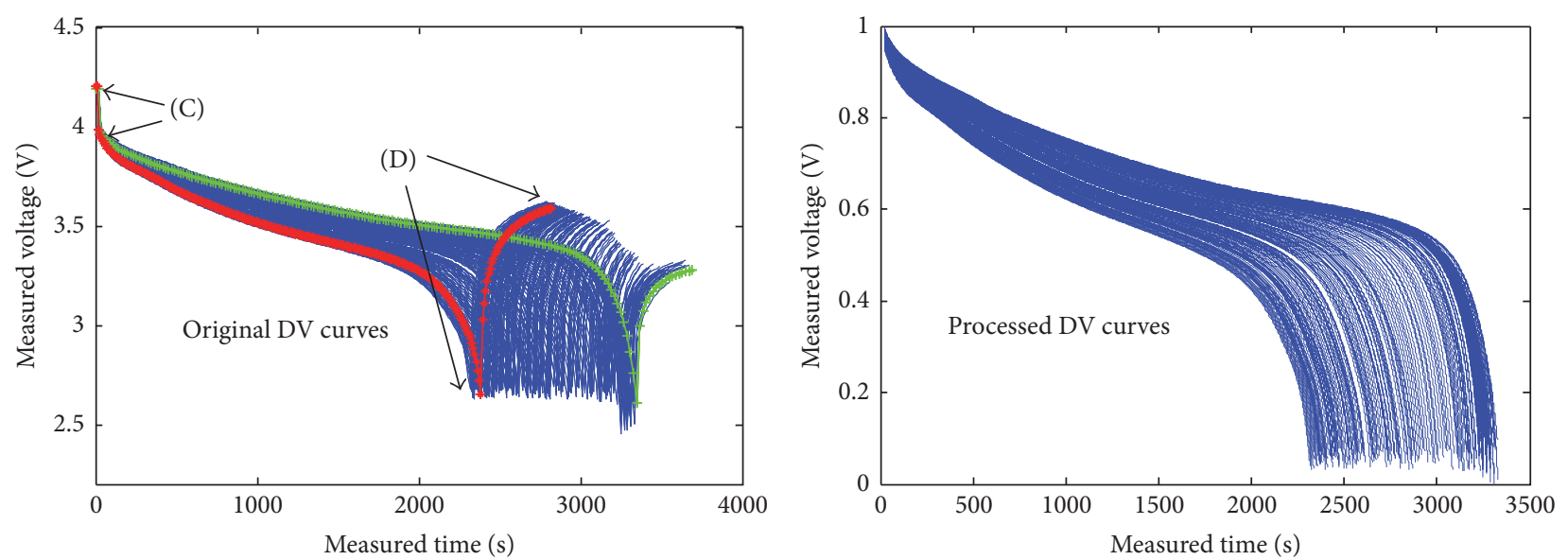

- The first curve

$\rightarrow$ The last curve

(c)

(d)

FIGURE 4: CC and DV curves: original versus processed. 
The difference in sampling rate (or sampling start time) results in a different number of CC or DV data from each cycle. This causes a problem on forming images of the same size. We adopt an interpolation method to ensure the same number of data points for each cycle. If an image is too large, the computational load becomes excessive, whereas if an image is too small, it cannot reflect the characteristics of the specific charge/discharge cycle. To balance these considerations, we select $M=N=64$. Thus, each cycle needs a total of 4096 data points to construct the image. If we obtain $i(i<4096)$ data points from a charge/discharge cycle, then the other 4096 - $i$ data points are acquired by the "spline" interpolation algorithm. Figure 4 shows an example of the processed CC/DV data curves from battery \#5.

\subsection{Feature Extraction Based on NSCT and LE}

3.3.1. NSCT-Based Multichannel Feature Extraction. This section describes a degradation feature extraction method based on NSCT, which is the core of this study. By utilizing NSCT, the transformed images from the CC/DV values are decomposed into multiple spatial-frequency channels with a set of orientation subbands. The subbands can be expressed as follows:

$$
\begin{aligned}
& \left\{C_{i_{0}}, C_{i, j}\right\}, \\
& i, i_{0}=1, \ldots, n ; i \leq i_{0} ; j=2,4,8, \ldots, m ; n \in N, m \in 2^{N},
\end{aligned}
$$

where $i$ is the decomposition scale, $j$ is the decomposition direction, $C_{i_{0}}$ represents the low-frequency coefficient, and $C_{i, j}$ represents the high-frequency coefficient of the $j$ th directional subband at the $i$ th scale. In this study, $i_{0}=2$ and $j=\{2,4\}$. That is, the decomposition scale is 2 and the decomposition directions at each scale are 2 and 4 .

The coefficients of the low-frequency subbands reflect the outline information of the image, whereas the coefficients of the high-frequency subbands reflect the detailed information. Therefore, three time-domain indicators are extracted as feature values; these are the mean value $(\mu)$ and the variance value $(\sigma)$ of the low-frequency subband coefficients as well as the energy value $(E)$ of the high-frequency subband coefficients. The equations for calculating these three indicators are given as follows:

$$
\begin{aligned}
& \mu=\frac{1}{M \times N} \sum_{x=1}^{M} \sum_{y=1}^{N} P(x, y) \\
& \sigma=\frac{1}{M \times N} \sum_{x=1}^{M} \sum_{y=1}^{N}[P(x, y)-\mu]^{2} \\
& E=\frac{1}{M \times N} \sum_{x=1}^{M} \sum_{y=1}^{N}[P(x, y)]^{2}
\end{aligned}
$$

where $P(x, y)$ represents each element of the coefficients and $M \times N$ represents the size of the coefficient matrix. Thus, an eight-dimensional feature vector of each image transformed from a single charge/discharge cycle can be obtained as

$$
f=\left[\mu, \sigma, E_{1,1}, E_{1,2}, E_{2,1}, E_{2,2}, E_{2,3}, E_{2,4}\right] \text {. }
$$

3.3.2. LE-Based Intrinsic Manifold Establishment. An intrinsic manifold is established using the aforementioned LE method. The degradation law governing the battery performance is revealed by data lying on this intrinsic manifold in the $M^{d}$ space, which is embedded in the highdimensional $R^{m}$ space. The $R^{m}$ space is constructed by the eight-dimensional feature vectors extracted by NSCT from the images transformed from the CC/DV data. The mapping $g=f^{-1}$ from $R^{m}$ to $M^{d}$ gives a $2 \mathrm{D}$ feature matrix in the $M^{d}$ space, where Li-ion battery capacity degradation can be well described. The mapping $g=f^{-1}$ is established by an analogous set of raw experimental data of full-cycle of lifetime (ASL) for each of the four typical datasets. Given an arbitrary point in $R^{m}$, the corresponding data point representing the Li-ion battery capacity in $M^{d}$ can be obtained through the mapping $g=f^{-1}$.

3.4. Capacity Estimation Based on Geodesic Distance. In this study, the geodesic distance along the intrinsic manifold between the initial point and the most recent point in the degradation process is calculated to carry out the estimation of battery capacity. Denote $C_{A_{0}}$ as the initial capacity, which is typically not the rated capacity, and $C_{\mathrm{EOL}}$ as the capacity of the final charge/discharge cycle of the ASL experimental data. We denote the geodesic distance between the initial point and the points on the intrinsic manifold $M^{d}$ as geo $_{s}$, and the geodesic distance between the initial point and the last point on the intrinsic manifold of the ASL as geo ${ }_{\mathrm{EOL}}$. The capacity of each point in $R^{m}$ space can then be estimated as

$$
\widehat{C}_{A}=C_{A_{0}}-\frac{\text { geo }_{s}}{\text { geo }_{\mathrm{EOL}}}\left(C_{A_{0}}-C_{\mathrm{EOL}}\right) .
$$

\section{Results and Discussion}

We use battery \#5 to demonstrate the effectiveness of the proposed approach. Figure 4 shows the original CC data curves during the charging process (Figure 4(a)) and the DV data curves during the discharging process (Figure 4(c)).

The corresponding processed curves derived from the stable stage of battery $\# 5$ are shown in Figures $4(\mathrm{~b})$ and $4(\mathrm{~d})$. Using the transformation scheme depicted in Figure 3, the normalized data for each cycle of the charging/discharging processes are transformed into an image. Figure 5 shows examples of the transformed images from a single cycle of the charging and discharging processes.

After the image transformation, the NSCT method is employed to extract features from the transformed images, thereby forming an eight-dimensional feature vector constructed by calculating the mean and variance of the lowfrequency subband coefficients and the energy of the highfrequency subband coefficients. Through the mapping $g=$ $f^{-1}$ from $R^{m}$ to $M^{d}$ established by LE, we construct the intrinsic manifold in the 2D space $M^{d}$. This describes the degradation law of battery capacity. Figure 6 shows the intrinsic manifold of battery \#5 embedded in the eightdimensional $R^{m}$ space constructed by features extracted from the DV data. 


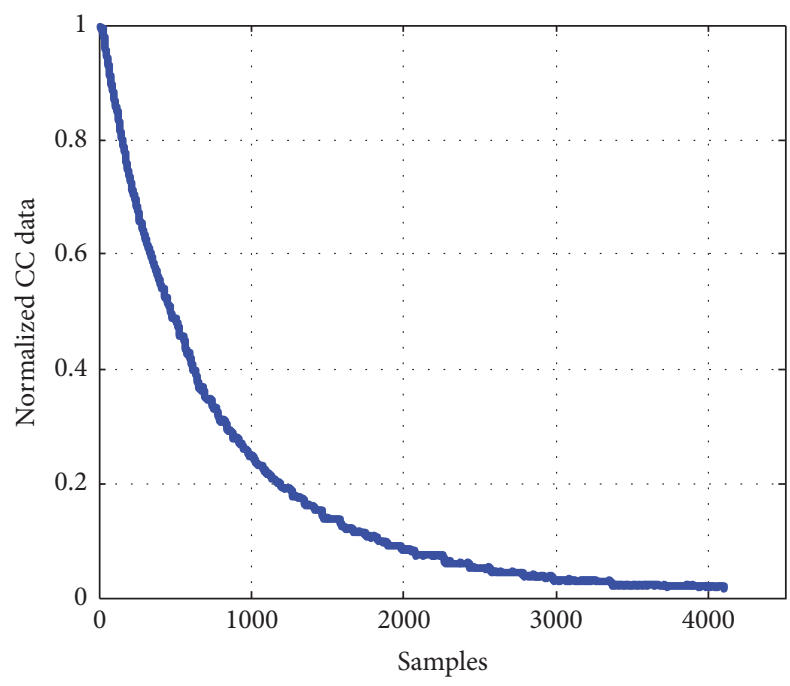

(a)

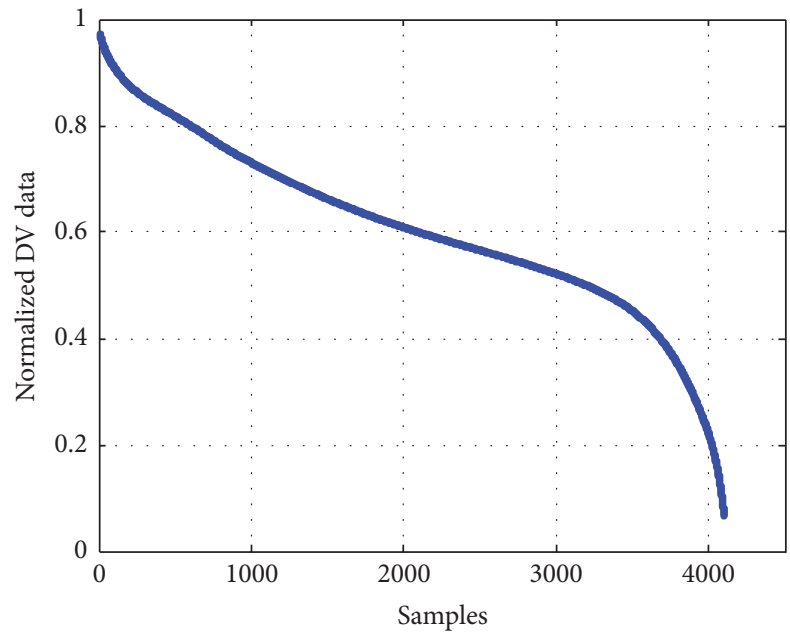

(c)

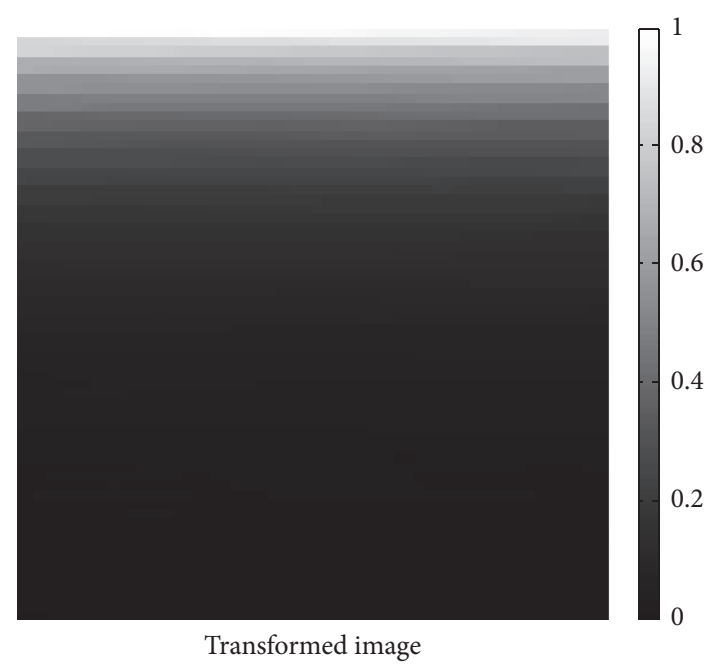

(b)

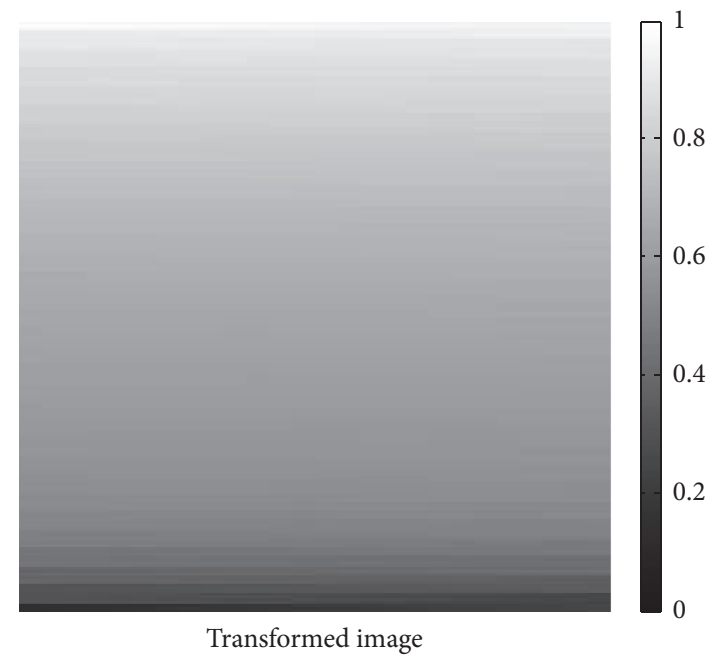

(d)

FIGURE 5: Image transformation based on CC/DV data from a single charging/discharging cycle.

In this study, the capacity is estimated using either CC or DV data based on the geodesic distance on the intrinsic manifold, as calculated by (14). The estimated results for all the demonstration data (batteries \#5, \#7, \#29, and \#54) under the different operating conditions closely track the measured capacity, as illustrated in Figure 7.

A comparison between the estimation results in this study and those of the study in $[16,17]$ is given in Table 2 in terms of the absolute error (AE), relative error (RE), and elapsed times (ETs). AE and RE are calculated as follows:

$$
\begin{aligned}
& \mathrm{AE}=\text { mean }[\mathrm{abs}(\text { Estimated capacity } \\
& \quad-\text { Estimated capacity })], \\
& \mathrm{RE} \\
& \quad=\frac{\text { abs (Estimated capacity }- \text { Estimated capacity })}{\text { Estimated capacity }} .
\end{aligned}
$$

Compared with [16], Table 2 shows that the proposed method based on visual cognition has approximate estimation accuracy with the similarity recognition method based on database. Using CC data for capacity estimation, the AEs and REs of batteries \#7 and \#29 based on the proposed method are smaller than those reported in [16], while the AEs and REs of batteries \#5 and \#54 are larger than those in [16]. Using DV data for capacity estimation, the AEs and REs of batteries \#29 and \#54 based on the proposed method are smaller than those in [16], while the AEs and REs of batteries \#5 and \#7 are larger than those in [16]. Even though the average AEs and REs of the proposed method are slightly larger than those in [16], however, the average ETs of the proposed method are only $11.1975 \mathrm{~s}$ based on CC data and $11.095 \mathrm{~s}$ based on DV data, more than 20 times smaller than those in [16], which makes the proposed visual cognition method more practical for real-time capacity estimation. 
TABLE 2: Estimation accuracy of available capacity based on the proposed method.

\begin{tabular}{|c|c|c|c|c|c|c|c|}
\hline Data & Items & $\# 5$ & $\# 7$ & $\# 29$ & $\# 54$ & Maximum & Average \\
\hline \multirow{3}{*}{$\mathrm{CC}$} & AEs (\%) & 2.30 & 2.51 & 0.90 & 3.18 & 3.18 & 2.222 \\
\hline & REs (\%) & 1.51 & 1.56 & 0.53 & 3.38 & 3.38 & 1.745 \\
\hline & ETs (s) & 16.16 & 15.86 & 3.59 & 9.18 & 16.16 & 11.1975 \\
\hline \multirow{3}{*}{$\begin{array}{l}\text { CC } \\
{[16]}\end{array}$} & AEs (\%) & 2.19 & 3.70 & 5.04 & 2.66 & 3.70 & 3.3975 \\
\hline & REs (\%) & 1.42 & 2.23 & 2.90 & 2.71 & 2.90 & 2.315 \\
\hline & ETs (s) & 401 & 291 & 11 & 189 & 401 & 223 \\
\hline \multirow{3}{*}{ DV } & AEs (\%) & 2.85 & 2.77 & 1.30 & 2.28 & 2.85 & 2.300 \\
\hline & REs (\%) & 1.76 & 1.69 & 0.75 & 2.43 & 2.43 & 1.658 \\
\hline & ETs (s) & 15.70 & 15.41 & 3.82 & 9.45 & 15.70 & 11.095 \\
\hline \multirow{3}{*}{$\begin{array}{l}\text { DV } \\
{[16]}\end{array}$} & AEs (\%) & 1.21 & 1.94 & 1.49 & 2.37 & 2.37 & 1.7525 \\
\hline & REs (\%) & 0.77 & 1.15 & 0.87 & 2.48 & 2.48 & 1.3175 \\
\hline & ETs (s) & 460 & 387 & 10 & 53 & 460 & 227.5 \\
\hline \multirow{2}{*}{$\begin{array}{l}\mathrm{CC}+\mathrm{DV} \\
{[17]}\end{array}$} & AEs (\%) & 4.48 & 2.42 & 1.85 & 3.71 & 4.48 & 3.115 \\
\hline & REs (\%) & 2.93 & 1.49 & 1.06 & 3.84 & 3.84 & 2.330 \\
\hline
\end{tabular}

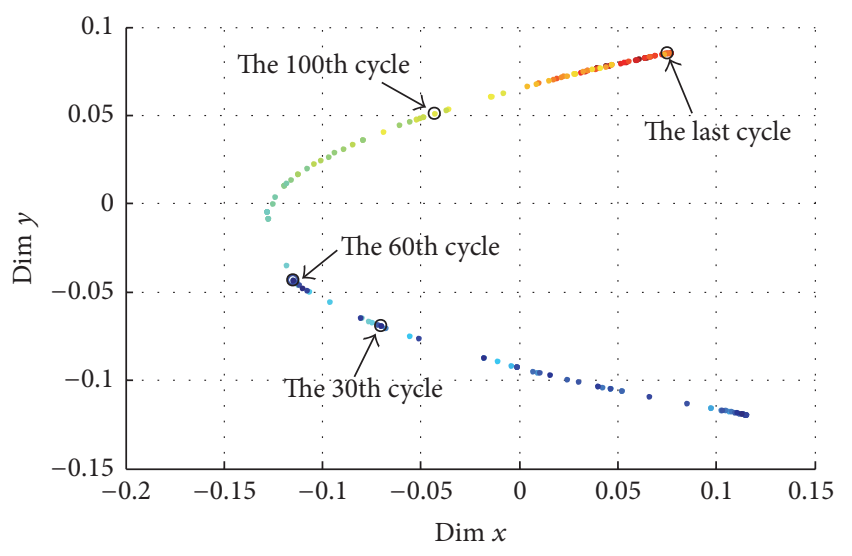

FIGURE 6: The intrinsic manifold of battery \#5 that reveals the battery performance degradation.

Compared with [17], it can be seen from Table 2 that the proposed capacity estimation method based on visual cognition generally exhibits better performance than the geometric-based method [17]. The AEs and REs for batteries $\# 5$, \#29, and \#54 are smaller than those reported in [17], with the AE and RE of battery \#7 being slightly higher. Notably, the excess part of the $\mathrm{AE}$ and $\mathrm{RE}$ of battery $\# 7$ is relatively small, because the estimation accuracy of battery \#7 in [17] is already very high. Using visual cognition based on CC data, the maximum and average of the AEs are reduced by $1.3 \%$ and $0.8925 \%$, respectively; those of the REs are reduced by $0.46 \%$ and $0.585 \%$, respectively. Similarly, using visual cognition based on DV data, the maximum and average of the AEs are reduced by $1.63 \%$ and $0.815 \%$, and those of the REs are reduced by $1.41 \%$ and $0.6725 \%$.

The estimation results presented in Figure 7 and Table 2 demonstrate that the proposed visual cognition-based capacity estimation method is highly effective with either CC or
DV data in a very short time. That is, one can choose either CC or DV curves with which to estimate the battery capacity in real time with high accuracy.

\section{Conclusions}

This study proposes a novel method for estimating the capacity of Li-ion batteries based on visual cognition. The proposed approach transforms the collected CC or DV data from each charge/discharge cycle into an image. NSCT is then employed to extract features from the transformed image. After that, taking inspiration from the HVS manifold sensing characteristic, we utilize the LE method to establish the intrinsic manifold embedded in the high-dimensional NSCT coefficients, from which the degradation law of battery performance can be revealed. The geodesic distance on the intrinsic manifold is adopted to estimate the battery capacity.

The proposed visual cognition-based capacity estimation method can use either CC or DV data. Verification experiments were conducted using data collected from the NASA battery data sets. The results demonstrate that the proposed method can be used to perform the capacity estimation using either CC or DV data with high accuracy under different operating and aging conditions. In addition, the proposed method avoids the need to study complex electrochemical mechanisms, establish models, or conduct lengthy testing, which makes it a promising practical method for battery capacity estimation. However, further research should be conducted to ascertain the following:

(1) the optimal number of CC/DV data for image transformation;

(2) the choice of decomposition scale and decomposition direction in the NSCT method;

(3) the intrinsic dimensionality of the manifold constructed by LE. 


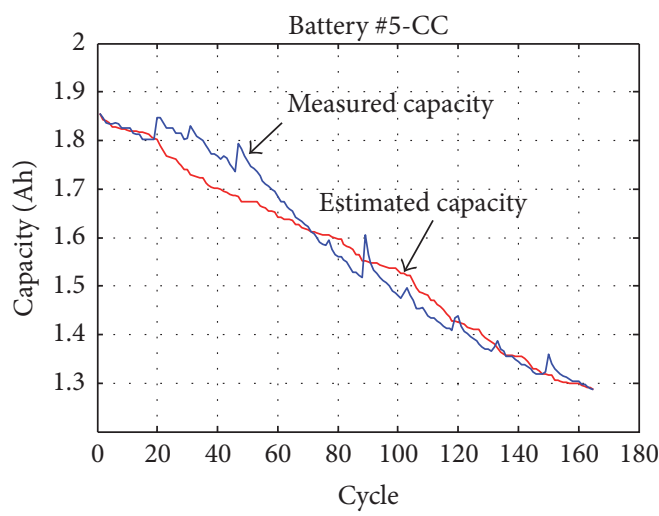

(a)

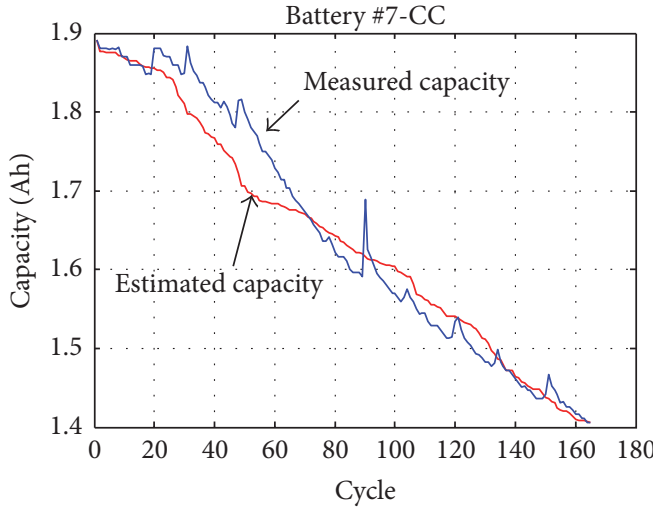

(c)

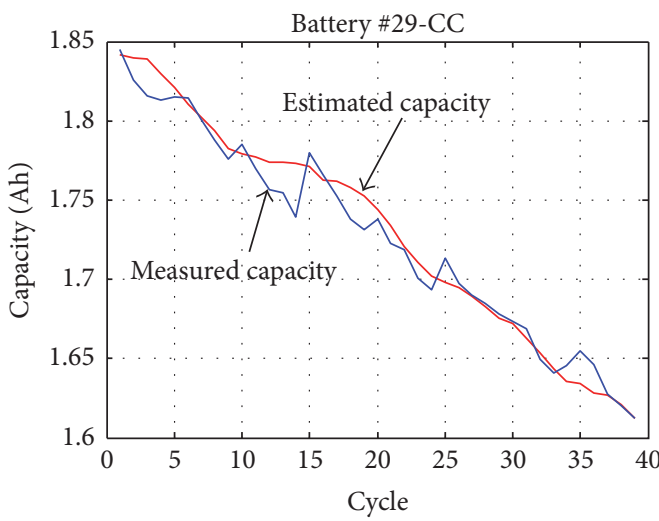

(e)

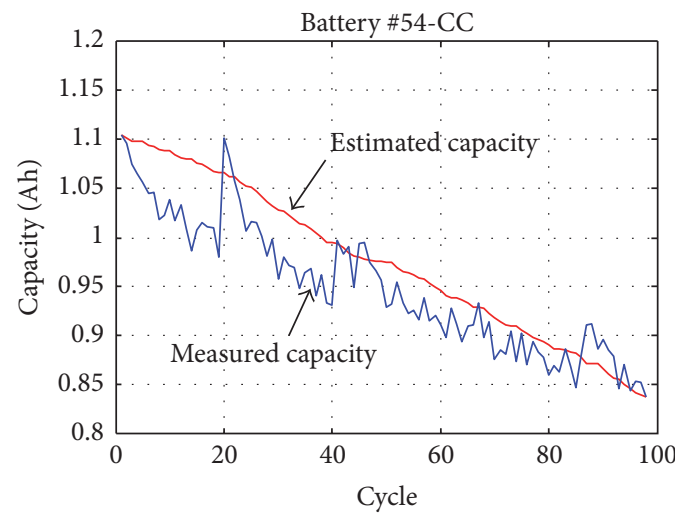

(g)

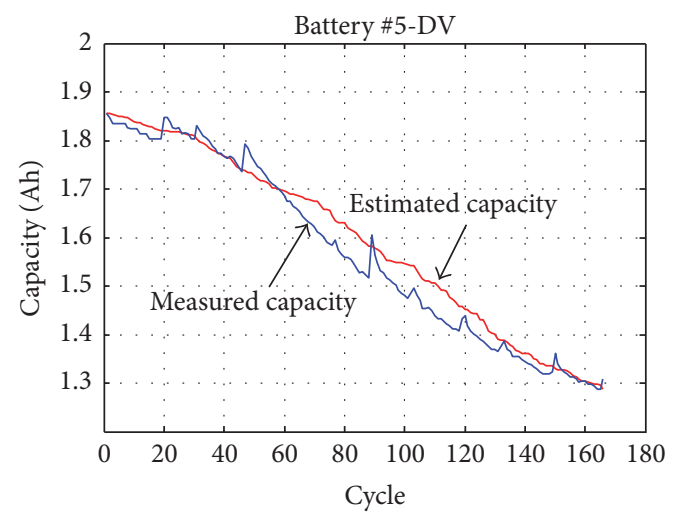

(b)

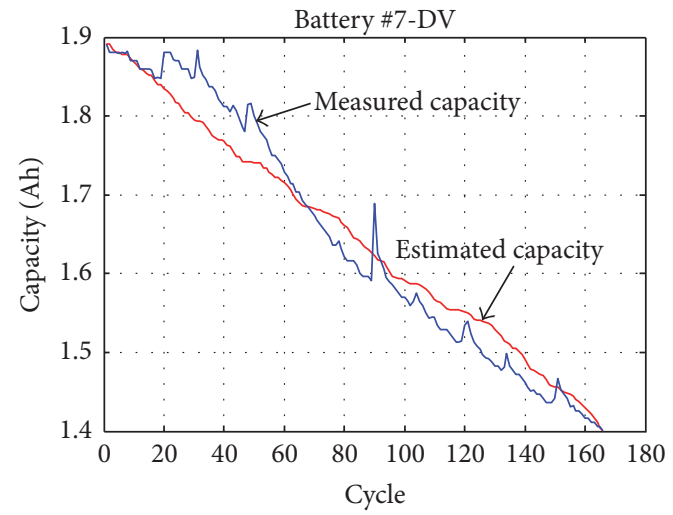

(d)

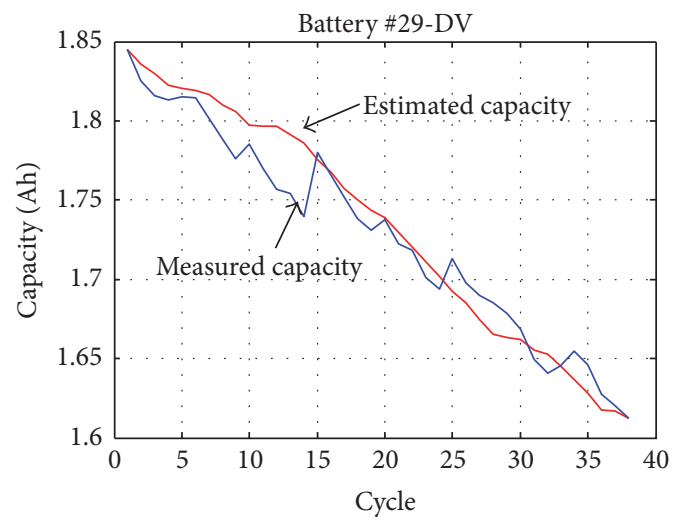

(f)

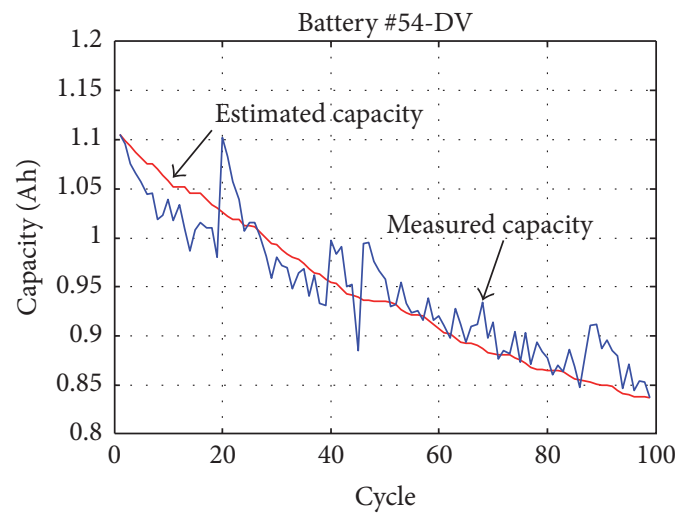

(h)

FIGURE 7: Capacity estimation results of typical data under various conditions based on CC/DV values. 


\section{Glossary}

AE: $\quad$ Absolute error

ASL: An analogous set of full-cycle of lifetime

AT: Ambient temperature

CC: Charging current

CT: $\quad$ Contourlet transform

CV: $\quad$ Constant voltage

DC: Discharge current

DFB: Directional filter bank

DV: Discharging voltage

EOD: End of discharge

EOLC: End-of-life criterion

HVS: Human visual system

IC: Initial capacity

LE: Laplacian eigenmap

LP: $\quad$ Laplacian pyramid

MCC: Multichannel characteristic

MSC: Manifold sensing characteristic

NSCT: Non-subsampled contourlet transform

NSDFB: Non-subsampled directional filter bank

NSFB: Non-subsampled filter bank

NSPFB: Non-subsampled pyramid filter bank

RE: Relative error.

\section{Conflicts of Interest}

The authors declare that there are no conflicts of interest regarding the publication of this article.

\section{Acknowledgments}

This study was supported by the Fundamental Research Funds for the Central Universities (Grant no. YWF-16-BJ-J18) and the National Natural Science Foundation of China (Grant nos. 51575021 and 61603016), as well as the China Postdoctoral Science Foundation (Grant nos. 2017M610033 and 2017T100026).

\section{References}

[1] L. Chen, W. Lin, J. Li, B. Tian, and H. Pan, "Prediction of lithium-ion battery capacity with metabolic grey model," Energy, vol. 106, pp. 662-672, 2016.

[2] G. Dong, X. Zhang, C. Zhang, and Z. Chen, "A method for state of energy estimation of lithium-ion batteries based on neural network model," Energy, vol. 90, pp. 879-888, 2015.

[3] B. Xia, C. Chen, Y. Tian, M. Wang, W. Sun, and Z. Xu, "State of charge estimation of lithium-ion batteries based on an improved parameter identification method," Energy, vol. 90, pp. 1426-1434, 2015.

[4] Z. Deng, L. Yang, Y. Cai, H. Deng, and L. Sun, "Online available capacity prediction and state of charge estimation based on advanced data-driven algorithms for lithium iron phosphate battery," Energy, vol. 112, pp. 469-480, 2016.

[5] L. Zheng, L. Zhang, J. Zhu, G. Wang, and J. Jiang, "Coestimation of state-of-charge, capacity and resistance for lithium-ion batteries based on a high-fidelity electrochemical model," Applied Energy, vol. 180, pp. 424-434, 2016.
[6] B. Y. Liaw, R. G. Jungst, G. Nagasubramanian, H. L. Case, and D. H. Doughty, "Modeling capacity fade in lithium-ion cells," Journal of Power Sources, vol. 140, no. 1, pp. 157-161, 2005.

[7] C. Hu, B. D. Youn, and J. Chung, "A multiscale framework with extended Kalman filter for lithium-ion battery SOC and capacity estimation," Applied Energy, vol. 92, pp. 694-704, 2012.

[8] R. Xiong, F. Sun, Z. Chen, and H. He, "A data-driven multi-scale extended Kalman filtering based parameter and state estimation approach of lithium-ion olymer battery in electric vehicles," Applied Energy, vol. 113, pp. 463-476, 2014.

[9] Z.- W. He, M.-Y. Gao, G.-J. Ma, Y.-Y. Liu, and S.-X. Chen, "Online state-of-health estimation of lithium-ion batteries using Dynamic Bayesian Networks," Journal of Power Sources, vol. 267, pp. 576-583, 2014.

[10] A. Singh, A. Izadian, and S. Anwar, "Model based condition monitoring in lithium-ion batteries," Journal of Power Sources, vol. 268, pp. 459-468, 2014.

[11] J. Yi, J. Lee, C. B. Shin, T. Han, and S. Park, "Modeling of the transient behaviors of a lithium-ion battery during dynamic cycling," Journal of Power Sources, vol. 277, pp. 379-386, 2015.

[12] J. Li, L. Wang, C. Lyu, L. Zhang, and H. Wang, "Discharge capacity estimation for Li-ion batteries based on particle filter under multi-operating conditions," Energy, vol. 86, pp. 638-648, 2015.

[13] M. A. Roscher, J. Assfalg, and O. S. Bohlen, "Detection of utilizable capacity deterioration in battery systems," IEEE Transactions on Vehicular Technology, vol. 60, no. 1, pp. 98-103, 2011.

[14] M. Einhorn, F. V. Conte, C. Kral, and J. Fleig, "A method for online capacity estimation of lithium ion battery cells using the state of charge and the transferred charge," IEEE Transactions on Industry Applications, vol. 48, no. 2, pp. 736-741, 2012.

[15] J. Zhang and J. Lee, "A review on prognostics and health monitoring of Li-ion battery," Journal of Power Sources, vol. 196, no. 15, pp. 6007-6014, 2011.

[16] L. Tao, C. Lu, and A. Noktehdan, "Similarity recognition of online data curves based on dynamic spatial time warping for the estimation of lithium-ion battery capacity," Journal of Power Sources, vol. 293, pp. 751-759, 2015.

[17] C. Lu, L. Tao, and H. Fan, "Li-ion battery capacity estimation: A geometrical approach," Journal of Power Sources, vol. 261, pp. 141-147, 2014.

[18] B. Balagopal and M.-Y. Chow, “The state of the art approaches to estimate the state of health (SOH) and state of function (SOF) of lithium Ion batteries," in Proceedings of the 13th International Conference on Industrial Informatics, INDIN 2015, pp. 13021307, UK, July 2015.

[19] https://en.wikipedia.org/wiki/Cognitive_science.

[20] P. Cavanagh, "Visual cognition," Vision Research, vol. 51, no. 13, pp. 1538-1551, 2011.

[21] Y. Cheng, Y. Hou, C. Zhao, Z. Li, Y. Hu, and C. Wang, "Robust face recognition based on illumination invariant in nonsubsampled contourlet transform domain," Neurocomputing, vol. 73, no. 10-12, pp. 2217-2224, 2010.

[22] Y. Chai, H. Li, and X. Zhang, "Multifocus image fusion based on features contrast of multiscale products in nonsubsampled contourlet transform domain," Optik - International Journal for Light and Electron Optics, vol. 123, no. 7, pp. 569-581, 2012.

[23] K. Hammouche, O. Losson, and L. Macaire, "Fuzzy aura matrices for texture classification," Pattern Recognition, vol. 53, pp. 212-228, 2016. 
[24] T. Tan, "Texture feature extraction via visual cortical channel modelling," in Proceedings of the Proceedings., 11th IAPR International Conference on Pattern Recognition. Vol. IV. Conference D: Architectures for Vision and Pattern Recognition, , pp. 607-610, The Hague, Netherlands.

[25] H. S. Seung and D. D. Lee, "The manifold ways of perception," Science, vol. 290, no. 5500, pp. 2268-2269, 2000.

[26] J. B. Tenenbaum, V. de Silva, and J. C. Langford, "A global geometric framework for nonlinear dimensionality reduction," Science, vol. 290, no. 5500, pp. 2319-2323, 2000.

[27] S. T. Roweis and L. K. Saul, "Nonlinear dimensionality reduction by locally linear embedding," Science, vol. 290, no. 5500, pp. 2323-2326, 2000.

[28] M. N. Do and M. Vetterli, "The contourlet transform: an efficient directional multiresolution image representation," IEEE Transactions on Image Processing, vol. 14, no. 12, pp. 2091-2106, 2005.

[29] D. H. Hubel and T. N. Wiesel, "Receptive fields, binocular interaction, and functional architecture in the cat's visual cortex," The Journal of Physiology, vol. 160, pp. 106-154, 1962.

[30] S. Lili, Y. Jiachen, and Z. Zhuoyun, "Stereo picture quality estimatiom based on a multiple channel HVS model," in Proceedings of the 2009 2nd International Congress on Image and Signal Processing, CISP'09, China, October 2009.

[31] A. L. da Cunha, J. Zhou, and M. N. Do, “The nonsubsampled contourlet transform: theory, design, and applications," IEEE Transactions on Image Processing, vol. 15, no. 10, pp. 3089-3101, 2006.

[32] Q. Zhang and B. Guo, "Multifocus image fusion using the nonsubsampled contourlet transform," Signal Processing, vol. 89, no. 7, pp. 1334-1346, 2009.

[33] T. Deng and W. Xie, "Granule-view based feature extraction and classification approach to color image segmentation in a manifold space," Neurocomputing, vol. 99, pp. 46-58, 2013.

[34] M. Belkin and P. Niyogi, "Laplacian eigenmaps and spectral techniques for embedding and clustering," Advances in Neural Information Processing Systems, vol. 14, pp. 585-591, 2002.

[35] D. L. Donoho and C. Grimes, "Hessian eigenmaps: locally linear embedding techniques for high-dimensional data," Proceedings of the National Acadamy of Sciences of the United States of America, vol. 100, no. 10, pp. 5591-5596, 2003.

[36] Z. Zhang and H. Zha, "Principal manifolds and nonlinear dimensionality reduction via tangent space alignment," SIAM Journal on Scientific Computing, vol. 26, no. 1, pp. 313-338, 2004.

[37] M. Belkin and P. Niyogi, "Laplacian eigenmaps for dimensionality reduction and data representation," Neural Computation, vol. 15, no. 6, pp. 1373-1396, 2003.

[38] http://en.wikipedia.org/wiki/Geodesic.

[39] G. Wen, L. Jiang, and J. Wen, "Using locally estimated geodesic distance to optimize neighborhood graph for isometric data embedding," Pattern Recognition, vol. 41, no. 7, pp. 2226-2236, 2008. 


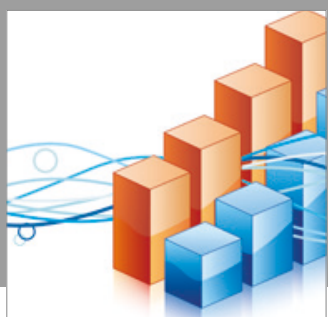

Advances in

Operations Research

vatersals

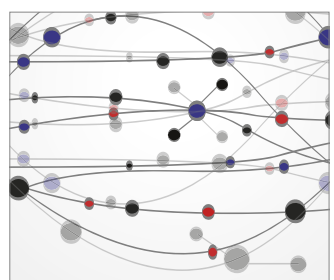

\section{The Scientific} World Journal
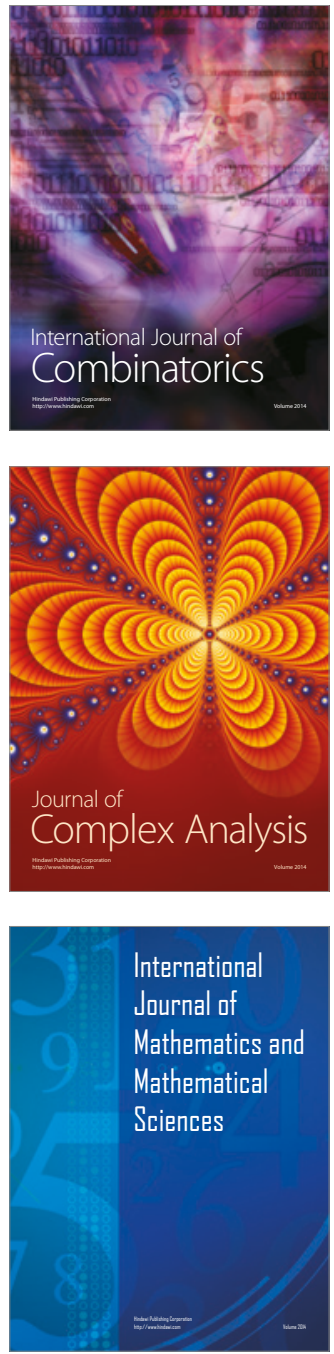
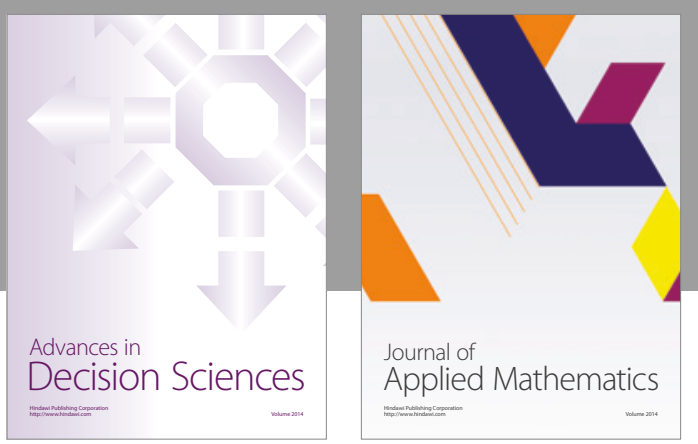

Algebra

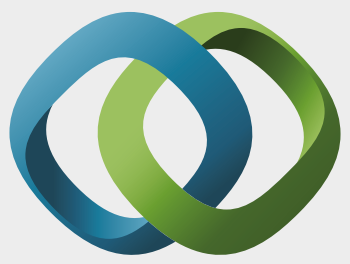

\section{Hindawi}

Submit your manuscripts at

https://www.hindawi.com
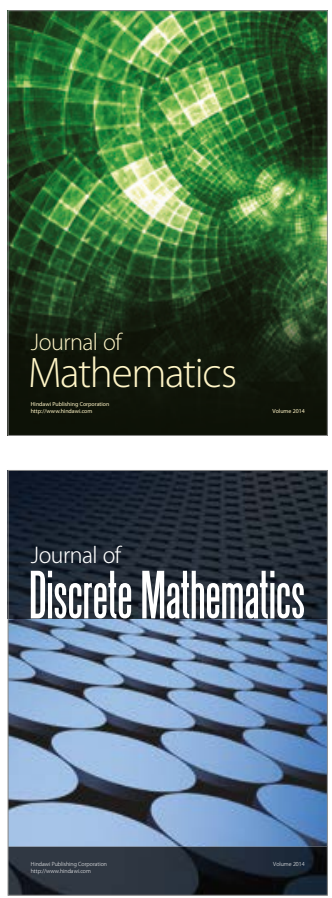

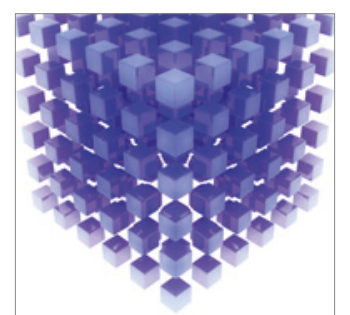

Mathematical Problems in Engineering
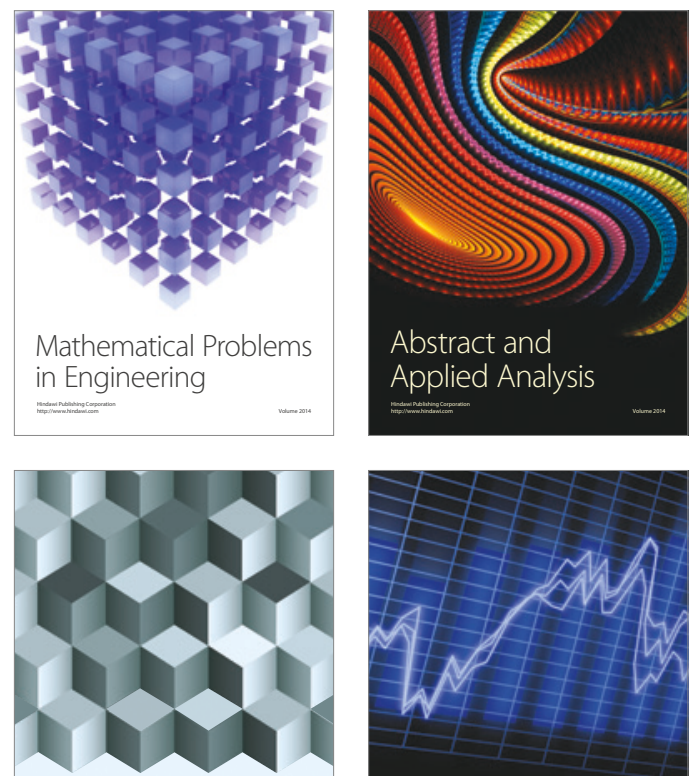

Journal of

Function Spaces

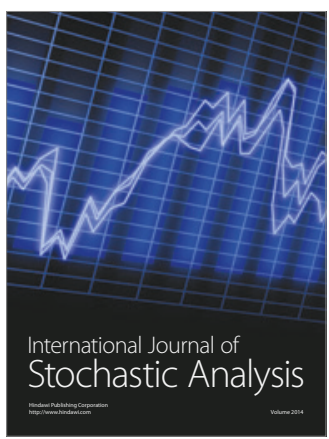

Probability and Statistics
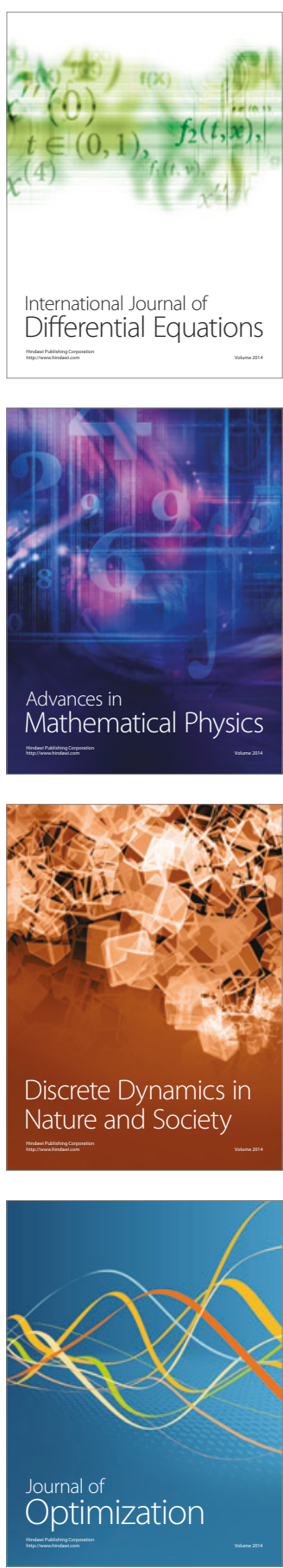